a
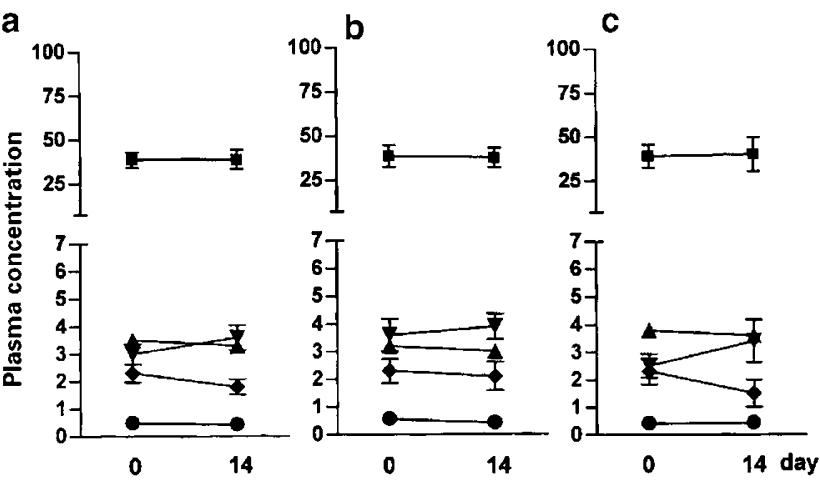

$\rightarrow$ DHEA

$\rightarrow-3 \alpha, 5 \alpha-T H P$

$\pitchfork$ Progesterone

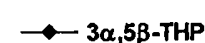

the ions that are characteristic for progesterone out of which the ion $m / z 178$ represents also pregnenolone which partially coelutes with progesterone. This explains why the progesterone values reported were too high. We now recalculated progesterone excluding $m / z$ 178. The corrected mean progesterone concentrations are below $5 \mathrm{nmol} / \mathrm{l}$ as usually found in the literature. The other steroid concentrations reported (Padberg et al, 2002) are correct. We seriously apologize for this error. Nevertheless, we would like to emphasize that the recalculated progesterone data do not change the conclusions of this study that treatment with rTMS for 2 weeks does not affect plasma concentrations of neuroactive steroids in depressive patients.

\section{REFERENCES}

Padberg F, di Michele F, Zwanzger P, Romeo E, Bernardi G, Schule $C$ et al (2002). Plasma concentrations of neuroactive steroids before and after repetitive transcranial magnetic stimulation (rTMS) in depression. Neuropsychopharmacology 27: 874-878.

Romeo E, Ströhle A, Spalletta G, di Michele F, Hermann B, Holsboer $\mathrm{F}$ et al (1998). Effects of antidepressant treatment on neuroactive steroid concentrations in major depression. Am J Psychiatry 155: 910-913.

\title{
Amisulpride vs Risperidone in Chronic Schizophrenia: Results of a 6-Month, Double-Blind Study
}

\author{
D Sechter, J Peuskens, O Fleurot, W Rein and Y Lecrubier the Amisulpride Study Group
}

Neuropsychopharmacology (2003) 28, 610-6II. doi:10.1038/s..npp. I300 I74

Correction to: Neuropsychopharmacology (2002) 27, 10711081.

The authors wish to apologize for errors made in the above paper. The corrected section of text is produced below.

\section{EFFICACY}

Except where stated, efficacy data are presented using the 'maintenance' population and the last-observation-carried forward (LOCF) procedure (all other analyses yielded similar results).

Amisulpride was demonstrated to be not inferior to risperidone with respect to the primary efficacy parameter.
The PANSS total score decreased markedly in both groups (from $91.1 \pm 13.0$ at baseline to $58.9 \pm 22.6$ after 6 months in the amisulpride group and from $92.5 \pm 12.2$ to $61.1 \pm 20.9$ in the risperidone group) (Figure 1). The noninferiority analysis showed that the decrease in the PANSS total score from baseline was not inferior with amisulpride as compared to risperidone (90\% two-sided confidence interval $[-5.6 ; 4.0])$. The two-sided $95 \%$ confidence interval also confirms the noninferiority of amisulpride compared with risperidone (Table 3). Similar results were observed in the ITT overall population (from $91.7 \pm 12.6$ at baseline to $64.8 \pm 25.0$ after 6 months in the amisulpride group and from $92.9 \pm 12.3$ to $66.8 \pm 24.1$ in the risperidone group, $90 \%$ two-sided confidence interval $[-5.4 ; 3.7])$. 